\title{
Mudanças no sistema tributário e no mercado de crédito e seus efeitos sobre a informalidade no Brasil
}

Nelson Leitão Paes Programa de Pós-Graduação em Economia do PIMES/UFPE, pesquisador do CNPq e da Escola de Administração Fazendária (ESAF) do Ministério da Fazenda

\section{Palauras-chave \\ informalidade, equilíbrio geral, tributação, custo do crédito.}

\section{Classificação JEL E51, H20,} $\mathrm{J} 21$.

\footnotetext{
Key words

informality, general

equilibrium, taxation,

credit cost.
}

JEL Classification E51,H20,

\section{Abstract}

Resumo

O objetivo deste artigo é analisar o impacto que a adoção de políticas tributárias ou creditícias tem sobre o nível de informalidade no Brasil, por meio de uma abordagem de equilíbrio geral computável. Os resultados mostraram que uma redução de 1\% nas alíquotas tributárias efetivas sobre o produto, a renda do trabalho ou a renda do capital ou, então, a diminuição do custo do crédito no mesmo percentual poderia reduzir o tamanho do setor informal e o emprego informal, sem, entretanto, diminuir a arrecadação. Das políticas consideradas, a mais efetiva foi a redução da tributação sobre a produção.
In this paper we analyze the impacts of some changes in tax and credit policies on informal business in Brazil. To do this, we use a computable general equilibrium approach. The main findings shows that a reduction in 1\% of tax rates on production or on labor income or capital income can reduce the size of the informal sector and the number of informal jobs, without reducing tax revenue. A more accurate result can be obtained with a reduction in the cost of credit instead of using lower tax rates. The most effective policy seems to be the reduction of taxes on production. 


\section{1_ Introdução}

A economia informal está presente em todo o mundo, e são fortes as evidências de que ela esteja crescendo (Schneider, 2000).

No Brasil, o setor informal responde por algo entre $30 \%$ e $40 \%$ do PIB,${ }^{1}$ e é no mercado de trabalho que a informalidade assume características mais dramáticas. Segundo Ramos (2002), nada menos do que 52,6\% dos empregos são informais. A existência de um setor informal dessa magnitude tem impactos significativos sobre diferentes aspectos da economia. Pelo lado fiscal, um elevado grau de informalidade significa evasão e perda de base tributária. Além disso, a informalidade também pode ter efeitos prejudiciais sobre a produção, afetando o nível e a qualidade dos empregos gerados e, consequentemente, a produtividade e o crescimento da economia.

Diante de um quadro tão preocupante, uma questão relevante para a política econômica é perscrutar o que determina essa baixa taxa de formalização e que medidas adotar para elevá-la. Segundo Fernandes et al. (2004), um dos argumentos mais recorrentes para explicar esse fato está relacionado à estrutura tributária do País. A carga tributária, como proporção do PIB, aumentou conside- ravelmente na década de 1990, passando de 25,2\% em 1991 para 32,82\% em 2004, sendo considerada demasiadamente elevada para o nível de desenvolvimento econômico nacional. Não apenas o nível da carga tributária é considerado importante, mas também sua composição, que afeta as oportunidades de emprego e de produção.

Além da questão dos tributos, um segundo ponto concentra as atenções do debate: o elevado custo do crédito no Brasil. Sabe-se que o país possui juros reais elevados para os padrões mundiais há mais de uma década e que o "spread" bancário também é extremamente alto. A combinação desses dois fatores (juros e impostos elevados) reduz a potencialidade da economia formal.

No entanto, avaliar os impactos de mudanças na estrutura tributária ou no mercado de crédito sobre o setor formal da economia não é um exercício simples. Existem grandes dificuldades, como as apontadas por Fernandes et al. (2004), ${ }^{2}$ para a utilização de abordagens empíricas ou econométricas.

Diante dessas restrições, uma alternativa importante é o uso de modelos de equilíbrio geral computável. Por meio dessa abordagem, na impossibilidade de se avaliarem quantitativamente mudan-
Schneider (2002), Loayza (1997) e Carneiro (1997), por exemplo.

2 Segundo tais autores, a abordagem empírica não é recomendada, pois ficaria restrita às mudanças passadas, que podem não servir para analisar as propostas atuais. Por outro lado, o uso da econometria tem as próprias restrições, como a falta de séries suficientemente longas para a utilização de modelos de séries temporais e problemas de identificação e endogeneidade para o caso de modelos longitudinais, que acabam por restringir também o uso de dados em painel. Além disso, mudanças tributárias não são tão frequentes e, quando ocorrem, tendem a afetar toda a economia no mesmo instante do tempo, acarretando dificuldades de identificação dos parâmetros relevantes do modelo. 
Ver, por exemplo, Altig et al. (2001) e Auerbach e Kotlikoff (1987). O "Global Economy Model" do FMI também é outro exemplo. ças tributárias que ainda não ocorreram, simulam-se tais alterações em uma economia artificial, garantindo que essa economia possua certas características, consideradas desejáveis, da economia real. Ademais, esse instrumental fornece a flexibilidade necessária para a inclusão de variadas propostas de alteração tributária, bem como permite avaliar, de forma qualitativa e quantitativa, o comportamento de importantes variáveis econômicas durante todo o processo de ajustamento ao novo ambiente tributário. Trata-se, portanto, de ferramenta largamente utilizada por governos, acadêmicos e organismos internacionais na prospecção e avaliação da situação fiscal. ${ }^{3}$

O objetivo deste artigo é estimar o impacto sobre o nível de informalidade no Brasil da adoção de determinadas políticas tributárias ou creditícias por meio de uma abordagem de equilíbrio geral, avaliando as respostas das variáveis relacionadas ao emprego, ao produto e à arrecadação tributária a modificações na política tributária e no mercado de crédito.

\section{Economia informal}

O entendimento das possíveis causas que determinam o tamanho e o funcio- namento do mercado informal, assim como a sua quantificação, é crucial para a política econômica, na medida em que os impactos dos diferentes instrumentos adotados pelos sistemas de regulação e de tributação sobre o desempenho da atividade econômica poderão ser diferentes na presença da informalidade. Portanto, a análise da frequência com que as atividades informais ocorrem e sua magnitude, e da sua interação com a economia formal, é essencial para uma efetiva atuação em termos de política econômica.

Segundo Name e Bugarin (2003), entre os principais fatores econômicos que causam o aumento da economia informal estão o crescimento da carga tributária (impostos, taxas, contribuições sociais, etc.), o aumento da regulação na economia oficial, especialmente no mercado de trabalho (custos de registro e manutenção de empregados formais) e o desemprego. Além de aspectos econômicos, há os de natureza sociológica e psicológica, como o declínio da percepção de justiça e lealdade para com as instituições públicas, a redução do índice de moralidade e a redução do índice de percepção da corrupção, que atuam complementarmente aos fatores econômicos, tendo em vista que diminuem o 
custo de oportunidade da escolha dos indivíduos para atuar na informalidade.

Em relação especificamente à tributação, a literatura ${ }^{4}$ indica que o aumento na tributação ou na contribuição para a seguridade social seria uma das principais causas do aumento da economia informal. Quanto maior a diferença entre o custo total do trabalho na economia oficial e os ganhos líquidos dos trabalhadores, maiores são os incentivos para se evitar tal diferença e ficar na economia informal.

No Brasil, a informalidade assume contornos dramáticos. Segundo Pastore (2000), nada menos do que $60 \%$ dos trabalhadores brasileiros estão à margem da Previdência Social. Esse resultado é reforçado por Ramos (2002), que calculou em 52,6\% o tamanho da informalidade no mercado de trabalho. No lado da produção, os números não são menos impressionantes. Loayza (1997) estima que o tamanho do setor informal no Brasil atingiu 37,8\% do PIB no período entre 1990-1993. Por sua vez, Carneiro (1997) apresenta estimativas da participação de atividades informais como percentagem do PIB por setor para 1988, sendo o setor de construção o que apresenta a participação mais elevada $(36 \%)$, com média em torno de $30 \%$.
Shepherd e Holden (1993), utilizando dados do IBGE, estimaram de forma residual as atividades dos autoempregados e empresas informais (37\% do PIB) para 1985. Em trabalho mais recente, embora com números bastante similares, Schneider (2002) calcula a informalidade no Brasil em 39,8\%.

Entre as principais causas do crescimento do setor informal brasileiro, Carneiro (1997) cita o protecionismo e as políticas intervencionistas, a pesada burocracia e o sistema tributário ineficiente. Pastore (2000) sugere que a complexidade do sistema tributário e trabalhista também estaria nesse rol. Reis e Ulyssea (2005), reportando resultados de um seminário interno do IPEA, reforçam a conclusão de que o sistema tributário brasileiro está no cerne das causas da informalidade.

Além desses aspectos, há alguns outros igualmente importantes, mas que ainda são pouco estudados: pode-se citar, por exemplo, o diferencial de preço que atrai consumidores para os produtos informais; a fragilidade da fiscalização, que se concentra basicamente nas grandes firmas; os enormes custos regulatórios, em especial os sanitários; e a própria organização do sistema tributário nacional, que, em função, por exemplo,
Ver Schneider (2000); Johnson, Kaufmann e ZoidoLobatón (1998a e 1998b) e Tanzi (1999), entre outros. 
Por exemplo, Fullerton (1982), Auerbach e Kotlikoff (1987), Araújo e Ferreira (1999), Altig et al. (2001) e Paes e Bugarin (2006), entre inúmeros outros. do diferencial de alíquotas tributárias no comércio interestadual, acaba favorecendo a sonegação e a informalidade.

Por outro lado, o Serviço Brasileiro de Apoio às Micro e Pequenas Empresas (Sebrae), em 2004, adicionou mais um componente às causas da informalidade: o mercado de crédito. Isso porque em quase todos os países uma das vantagens de se ter uma empresa formal é justamente o acesso ao mercado de crédito. Entretanto, não é isso o que ocorre no Brasil. Segundo a pesquisa, 61\% das pequenas e microempresas brasileiras jamais tomaram um empréstimo bancário. A principal razão para a falta de acesso aos empréstimos, de acordo com os pesquisados, são as elevadas taxas de juros vigentes no País.

Nesse sentido, serão analisados dois aspectos fundamentais como determinantes da informalidade. Seguindo as conclusões da literatura que mostram forte relação entre carga tributária e formalização na economia, será avaliada quantitativamente a influência da tributação e da coerção para o cumprimento das obrigações tributárias sobre o tamanho do setor informal. Em seguida, procurar-se-á avaliar os efeitos dos custos de empréstimos sobre a escolha das firmas entre a economia oficial e a informal. A hipótese aqui é a de que, ao se reduzir o benefício das firmas formais, dificultando o acesso ao crédito bancário, se estimula o setor informal da economia.

\section{3_Modelo}

De forma a avaliar algumas sugestões de políticas de redução da informalidade, recorreu-se a um modelo de equilíbrio geral computável dinâmico. A economia artificial analisada baseia-se no modelo neoclássico de acumulação de capital com a utilização de tempo discreto. A economia é fechada, determinística, com população e tecnologia constantes. Supõe-se informação perfeita por parte de todos os agentes econômicos.

O uso de modelos de crescimento neoclássico é corrente na literatura econômica voltada para a análise de efeitos de mudanças tributárias. Um dos pioneiros em aplicações nessa área foi o artigo de Shoven e Whalley (1992) que trata de um modelo de equilíbrio geral aplicado na análise de questões relativas à reforma tributária norte-americana. De fato, são inúmeros os trabalhos na literatura nacional e internacional simulando alterações tributárias com base em modelos neoclássicos. ${ }^{5}$ Entretanto, o modelo usado nesse artigo explora questões relacio- 
nadas às implicações da tributação sobre a formalização da economia, o que representa um dos subconjuntos de aplicações em finanças públicas do modelo neoclássico. ${ }^{6}$ Por outro lado, trazer o acesso ao crédito na análise da dualidade formal/informal no âmbito do modelo neoclássico é o objetivo dos trabalhos de Straub (2005) e Antunes e Cavalcanti (2003 e 2007). Em particular, estes últimos autores adotam uma separação entre firmas formais e informais com uma abordagem dinâmica, similar a que será utilizada neste artigo.

As famílias, de vida infinita, são homogêneas e modeladas como uma família representativa que fornece mão de obra e capital para as firmas. Estas, em troca, pagam salários e juros. Tal família representativa aloca o seu tempo entre consumo e lazer de maneira a maximizar o seu fluxo descontado de utilidade, sujeito à sua restrição orçamentária. A renda das famílias é consumida ou poupada, sendo a poupança representada no modelo pelo capital físico.

O setor produtivo é modelado por duas firmas representativas, uma formal e outra informal, ambas produzindo o único bem dessa economia. A produção desse bem é destinada às famílias, e a receita é utilizada para o pagamento de salá- rios e remuneração do capital fornecido pelas famílias e financiadores externos. Apenas a firma formal é tributada. A firma informal e as famílias não pagam qualquer tipo de tributo.

A firma informal é competitiva, ao passo que a formal possui certo poder de mercado. Somente as firmas formais pagam tributos, que incidem sobre o valor da produção, do rendimento do trabalho e dos lucros. A firma formal tem acesso ao crédito, concedido por financiadores externos, a um custo menor do que o capital próprio fornecido pelas famílias. O setor informal, por sua vez, não paga nenhum tipo de imposto e não tem nenhum acesso ao crédito. Por outro lado, as firmas informais estão sujeitas à atuação dos órgãos de fiscalização de tributos, podendo ser multadas se forem descobertas. $^{7}$ Observa-se que se tentou captar no modelo as vantagens e desvantagens da formalização - a sonegação, o custo e o acesso ao crédito, a estrutura de mercado diferenciada e o risco de autuações.

O governo arrecada tributos das firmas formais, multa as firmas informais, faz transferências para as famílias e gasta com o fornecimento de bens públicos, mantendo um orçamento equilibrado em cada período.

\footnotetext{
Entre os vários trabalhos, citamos Piggot e Whalley (2001), Amaral e Quintin (2006) e Fernandes et al. (2004).

7 Neste ponto estão presentes algumas ideias importantes a respeito do sistema institucional-legal, uma vez que, quanto maior for a vigilância das autoridades tributárias e mais eficiente for o Poder Judiciário em fazer cumprir as multas e penalidades impostas, menor o incentivo para as firmas permanecerem na informalidade.
} 
A apresentação matemática do modelo com todas as suas equações, as definições do equilíbrio e o método numérico aplicado na sua solução estão no Apêndice.

\section{4_Calibragem}

A calibragem dos parâmetros envolveu dados da Pesquisa de Orçamentos Familiares (POF) 2002/2003, do Censo 2000 e do Novo Sistema de Contas Nacionais - todas do IBGE -, além da base de dados do IPEA (IPEADATA) e de relatórios gerenciais da Secretaria da Receita Federal (SRF). Normalizou-se o produto da economia como $Y=1$. Nesta seção, busca-se determinar o estado da economia brasileira em 2004, representado por uma série de parâmetros e variáveis relevantes.

\section{1_Contas Nacionais}

Usando dados do Novo Sistema de Contas Nacionais do IBGE, observa-se que o rendimento do trabalho no ano de 2004 correspondeu a somente 46,45\% da renda agregada da economia brasileira. Do lado da demanda, as Contas $\mathrm{Na-}$ cionais distribuem-se entre consumo (62,19\% do PIB), investimento (17,81\% do PIB) e governo (20,00\% do PIB). Do lado da renda, imposto sobre a produção $\left(a_{P}\right)$ fica com $16,28 \%$ do PIB, enquanto a renda do capital e a renda do trabalho dividem o restante. Já os tributos sobre a renda do trabalho respondem por uma arrecadação de $10,19 \%$ do $\operatorname{PIB}\left(a_{h}\right)$, e os sobre a renda do capital, por 6,36\% do PIB $\left(a_{k}\right)$.

\section{2_ Distribuição do produto entre o setor formal e o informal}

Segundo a literatura, são muitas as estimativas do tamanho do setor informal brasileiro com valores próximos entre 25\% e 40\% do PIB. Loayza (1997) e Carneiro (1997) estimam em 37\% e $30 \%$, respectivamente; Antunes e Cavalcanti (2003), em 35\%; e Schneider e Enste (2000), entre $25 \%$ e $35 \%$. Optou-se por um valor médio entre essas estimativas, adotando-se uma fatia de $30 \%$ do produto para o setor informal e $70 \%$ para o setor formal.

\section{3_ Taxa de juros, "spread" e oferta de crédito}

A primeira hipótese básica é a de que a remuneração do capital de terceiros, $\mathrm{r}_{\mathrm{t}}-\theta$, equivale ao valor da taxa Selic em dezembro de 2004, isto é, $17,75 \%$. Em seguida, supomos que o custo do capital de terceiros equivale a $75 \%$ da remuneração do capital próprio, $\mathrm{r}_{\mathrm{t}}$ (ANEEL, 2001). 
Dadas essas hipóteses, e sabendo que a taxa de inflação no período medida pelo IPCA foi de $6,05 \%$, calculamos $\mathrm{r}=15,60 \%$ e $\theta=3,90 \%$.

Em relação ao tamanho do crédito, segundo dados do Relatório Anual do Banco Central do Brasil em 2004, o volume total de empréstimos na economia foi de $26,2 \%$ do PIB, sendo $72 \%$ para as pessoas jurídicas e $28 \%$ para as pessoas físicas. Dado que, no modelo, se trabalha com crédito para as empresas, o volume total de crédito ofertado para as firmas é de $b=0,1877$.

\section{4_ Parâmetro para o custo da informalidade para as empresas informais}

Segundo dados da Receita Federal do Brasil, disponíveis no sítio da instituição, em 2004 foram aplicados multas no total de $\mathrm{R} \$ 65.772$ milhões, o equivalente a $3,39 \%$ do PIB. Como a arrecadação no período foi de $32,82 \%$ do PIB, reduziu-se o valor da arrecadação a ser obtida das empresas formais do valor arrecadado das multas, restando, portanto, 29,43\% do PIB a ser arrecadado.

Assim, o custo da sonegação para as empresas informais pode ser obtido igualando-se o total de multas aplicadas com o custo calculado pelo modelo:

$$
\mathrm{pk}_{\mathrm{It}}^{\alpha} \mathrm{h}_{\mathrm{tt}}^{\beta}+\mathrm{pw}_{\mathrm{t}} \mathrm{h}_{\mathrm{It}}+\mathrm{pr}_{\mathrm{t}} \mathrm{k}_{\mathrm{it}}=0,0339 \Leftrightarrow \mathrm{p}=\frac{0,0339}{2 \mathrm{Y}_{\mathrm{I}}}
$$$$
\text { O que nos fornece } \mathrm{p}=5,65 \%{ }^{8}
$$

\section{5_Salário}

O cálculo do salário é obtido equiparando-se o rendimento líquido do trabalho com a participação da renda do trabalho nas Contas Nacionais, descontando-se a tributação sobre a renda do trabalho:

$\mathrm{wh}_{\mathrm{F}}+\mathrm{wh}_{\mathrm{i}}=50 \%\left(\mathrm{Y}-\mathrm{a}_{\mathrm{p}}\right)-\mathrm{a}_{\mathrm{h}} \Leftrightarrow \mathrm{w}=\frac{50 \%\left(\mathrm{Y}-\mathrm{a}_{\mathrm{p}}\right)-\mathrm{a}_{\mathrm{h}}}{\mathrm{h}}$

Adotando-se $\mathrm{h}=0,3333$, o que pressupõe uma jornada de 8 horas de trabalho diária, temos que $\mathrm{w}=0,8612$.

\section{6_ Variáveis informais}

De posse dos parâmetros até agora calculados, pode-se obter as variáveis que compõem o setor informal da economia. Pelas equações (7) e (8) no Apêndice, calcula-se a participação da renda do capital informal no produto informal, $\alpha=0,3617$. Em seguida, obtém-se o estoque de capital informal, $\mathrm{k}_{\mathrm{I}}=0,6213$ e as horas de trabalho informais, $\mathrm{h}_{\mathrm{I}}=0,1986$. 
Segundo trabalho de Pinheiro e Miranda (2000), quase $60 \%$ dos brasileiros ocupados não possuem vínculos com a Previdência Social.

10 Apesar de o valor parecer muito alto, deve-se inicialmente ressaltar que se trabalhou com o salário líquido.

Normalmente a alíquota tributária incide sobre o salário bruto. Transformando essa alíquota em alíquota efetiva incidente sobre o salário bruto, encontra-se $44,35 \%$, valor muito mais próximo ao real. Deve-se lembrar que, sobre o salário pago pelas firmas formais, incidem a cobrança previdenciária - $20 \%$ do empregador, $8 \%$ a $11 \%$ do empregado, 5,8\% de Sesc, Sesi, Senac, Senai, Sebrae, FNDE, Incra e outros, e 3\% de seguro contra acidentes de trabalho - e o imposto de renda na fonte (alíquota efetiva média de $7,6 \%$ ) e FGTS, $8 \%$, o que já resulta em valores bastante próximos ao encontrado.

\section{7_ Variáveis formais e tributação}

\subsection{1_ Horas de trabalho formais}

Para o cálculo das horas de trabalho formais, usamos a condição de equilíbrio no mercado de trabalho, (11), no Apêndice, o que nos fornece $h_{F}=0,1347$. Esse resultado reflete os resultados de pesquisas recentes sobre a contratação de mão de obra formal, que indicam que 60\% das contratações são realizadas sem carteira assinada.

\subsection{2_ Produtividade do trabalho das empresas formais}

Conforme estudo da consultoria McKinsey e Company (2004), a produtividade do trabalho nas firmas informais correspondeu a um valor próximo de $40 \%$ da produtividade nas firmas formais. Fixando a produtividade das informais em 1 , encontramos $\mathrm{A}=2,5$.

\subsection{3_ Tributação sobre a produção}

Nesta categoria, estão representados todos os tributos ligados à produção e à importação, como o ICMS, o PIS, a COFINS, a CPMF, o ISS e o IPI, além de parte da CPMF, representando no total $16,28 \%$ do PIB. A alíquota efetiva é calculada, descontando-se o valor das multas: $\tau_{\mathrm{p}} \mathrm{Y}_{\mathrm{F}}+\mathrm{p} \mathrm{Y}_{\mathrm{I}}=\mathrm{a}_{\mathrm{p}} \Leftrightarrow \tau_{\mathrm{p}}=\frac{\mathrm{a}_{\mathrm{p}}-\mathrm{p} \mathrm{Y}_{\mathrm{I}}}{\mathrm{Y}_{\mathrm{F}}}$

Dados os valores já conhecidos, obtém-se $\tau_{\mathrm{k}}=20,84 \%$.

\subsection{4_ Tributação sobre a renda do trabalho}

Para a determinação da alíquota tributária sobre a renda do trabalho, foram utilizados os dados de arrecadação, descontadas as multas, o salário e as horas já calculados. Assim:

$\left(1+\tau_{\mathrm{h}}\right) \mathrm{wh}_{\mathrm{F}}+\mathrm{pwh}_{\mathrm{i}}=\mathrm{a}_{\mathrm{h}} \Leftrightarrow \tau_{\mathrm{h}}=\frac{\mathrm{a}_{\mathrm{h}}-\mathrm{pwh}_{\mathrm{I}}}{\mathrm{wh}_{\mathrm{F}}}$

Substituindo os valores, tem-se $\tau_{\mathrm{h}}=79,47 \%{ }^{10}$.

\subsection{5_ Tributação sobre a renda do capital}

O imposto sobre a renda do capital representou 6,36\% do PIB em 2004. O valor da alíquota pode ser calculado abaixo:

$$
\begin{gathered}
\tau_{\mathrm{k}}\left[\left(1-\tau_{\mathrm{p}}\right) \mathrm{Y}_{\mathrm{F}}-\left(1+\tau_{\mathrm{h}}\right) \mathrm{wh}_{\mathrm{F}}\right]+\mathrm{prk}_{\mathrm{i}}=\mathrm{a}_{\mathrm{k}} \\
\Leftrightarrow \tau_{\mathrm{k}}=\frac{\mathrm{a}_{\mathrm{k}}-\mathrm{prk}_{\mathrm{I}}}{\left(1-\tau_{\mathrm{p}}\right) \mathrm{Y}_{\mathrm{F}}-\left(1+\tau_{\mathrm{h}}\right) \mathrm{wh}_{\mathrm{F}}}
\end{gathered}
$$

Calculando-se, tem-se $\tau_{\mathrm{k}}=16,79 \%$

\subsection{6_ Parâmetros da função de produção formal - percentual do capital total usado para 0 fornecimento de crédito - estoque de capital formal - lucro}

$\mathrm{O}$ parâmetro $\gamma_{\mathrm{F}}$ pode ser obtido da equação (10) do Apêndice em conjunto com a definição da função de produção formal, $Y_{F}=\left(k_{F}+b\right)^{\alpha_{F}}\left(A h_{F}\right)^{\gamma_{F}}$ o que nos for- 
nece $\gamma_{\mathrm{F}}=0,3757$. Já a os parâmetros $\alpha_{\mathrm{F}}$ e $\eta$, que medem o percentual do estoque do capital formal que é aceito como colateral no crédito, são calculados simultaneamente, utilizando-se a equação (17) do Apêndice e a definição $\eta=\mathrm{b} / \mathrm{k}_{\mathrm{F}}$, obtendo-se $\alpha_{\mathrm{F}}=0,3733 \mathrm{e}$ $\eta=0,1951$. Finalmente, $\mathrm{o}$ estoque de capital pode ser calculado diretamente da função de produção formal, $\mathrm{k}_{\mathrm{F}}=0,9625$, e o lucro da firma formal da equação (13) do Apêndice, $\pi_{\mathrm{F}}=0,1157$.

\section{8_ Estoque de capital das famílias}

Somando-se o valor do capital nas firmas formais e informais, obtém-se o total de capital das famílias empregado na economia, $\mathrm{k}=\mathrm{k}_{1}+\mathrm{k}_{\mathrm{F}}=1,5838$.

\section{9_ Така de desconto intertemporal, peso do consumo na função utilidade e depreciação}

Utilizando-se a informação relativa ao investimento privado e a própria definição do investimento em estado estacionário, encontra-se a depreciação, por meio da equação (3) no Apêndice, $\delta=0,1098$. Da equação da dinâmica do consumo em estado estacionário, (5), no Apêndice, encontra-se a taxa de desconto intertemporal, $\beta=0,956$. Por último, calcula-se o peso que as famílias dão ao consumo na função utilidade com a equação (4), no Apêndice, em estado estacionário, $\phi=0,5144$.

\subsection{0_Transferências governamentais}

As transferências para as famílias são calculadas por meio da restrição orçamentária (2) no estado estacionário, o que nos fornece $\mathrm{T}=0,1326$.

\section{5_Propostas}

As propostas para políticas de redução da informalidade que serão examinadas neste trabalho referem-se basicamente à tributação e ao mercado de crédito. Serão simulados seis tipos de políticas implementadas de imediato, sendo o ano de 2004 correspondente ao período inicial, como descritas a seguir:

i. Medidas de politica tributária - contemplam separadamente a redução de $1 \%$ nas alíquotas da tributação sobre o consumo, renda do capital e renda do trabalho. O objetivo aqui é verificar a magnitude da influência da tributação sobre o grau de formalização da economia.

ii. Aumento do custo da informalidade em 1\%. Neste caso, há um aumento no custo percebido pela firma informal em permanecer na informalidade. Esse aumento de 
custo pode decorrer de vários fatores, tais como:

a. melhoria do aparato tecnológico do órgão fiscalizador;

b. aumento das penalidades associadas à sonegação;

c. aumento da probabilidade percebida pelas firmas informais de serem fiscalizadas;

d. melhoria na eficiência e na rapidez do Judiciário em fazer cumprir a aplicação das penalidades tributárias.

iii. Medidas de politica de crédito - Redução do custo do capital de terceiros em 1\% ou aumento da disponibilidade de crédito, pelo incremento do parâmetro $\eta$ em 1\%. No primeiro caso, o custo do empréstimo para as firmas formais fica mais barato. Como parte desse é devido a tributos, pode-se pensar aqui numa redução da cunha fiscal que encarece o custo do empréstimo. No segundo caso, há um aumento da disponibilidade de crédito para as empresas formais.

Das seis propostas acima, cinco têm caráter positivo, uma vez que não buscam reprimir a informalidade, e sim incentivar a formalização. Além disso, contêm ideias que também estimulam a atividade econômica e, possivelmente, trarão efeitos benéficos para toda a economia. A única proposta com caráter negativo, ou, mais corretamente, repressivo, é a que aumenta o custo da sonegação para as empresas informais, e que não traz maiores incentivos à economia.

As propostas serão avaliadas não apenas com relação aos seus efeitos sobre o grau de formalização, mas também quanto a outras variáveis importantes como a arrecadação.

\section{6_Resultados}

\section{1_Produto}

Realizadas as simulações, a taxa de variação do produto no novo estado estacionário, em relação à situação em 2004, pode ser vista na Tabela 1.

Os resultados sugerem que a política tributária é mais efetiva do que as demais. Percebe-se que diminuição de $1 \%$ na tributação gera forte redução do setor informal e um importante crescimento no setor formal. Em relação ao produto formal, são dois os fatores que o impulsionam:

i. migração de empresas para a formalidade, com aumento da produtividade média; 
Tabela 1_Variação do produto

\begin{tabular}{l|c|c|c}
\multicolumn{1}{c|}{ Propostas } & \multicolumn{2}{c}{ Variação (\%) } \\
\cline { 2 - 4 } & Informal & Formal & Total \\
\hline Redução da Tributação sobre a Produção & $-1,32$ & 0,79 & 0,15 \\
\hline Redução da Tributação sobre a Renda do Capital & $-0,44$ & 0,30 & 0,08 \\
\hline Redução da Tributação sobre a Renda do Trabalho & $-1,26$ & 0,67 & 0,09 \\
\hline Aumento do Custo da Informalidade & $-0,81$ & 0,27 & $-0,06$ \\
\hline Redução do Custo do Crédito & $-0,33$ & 0,19 & 0,03 \\
\hline Aumento da Oferta de Crédito & $-0,08$ & $-0,01$ & $-0,03$ \\
Fonte: Elaboraçãa própria.
\end{tabular}

ii. crescimento da produção originalmente formal, em função da redução da tributação ou dos custos financeiros. Mesmo somados esses dois efeitos, o crescimento do produto formal tem crescimento inferior ao decréscimo do produto informal, que, dada a maior participação e produtividade do primeiro, mantém o produto quase estável, com leve crescimento. A política de crédito tem efeitos limitados em razão da pequena participação do crédito em relação ao estoque de capital formal.

Medidas repressivas, como o aumento do custo da informalidade, também têm efeito importante sobre o setor informal, mas não há repercussão no crescimento da economia, visto que não há estímu- lo para o crescimento intrínseco do setor formal. Ocorre apenas uma migração de empresas informais para o setor formal.

A trajetória dinâmica do produto pode ser visualizada na Figura 1, adiante, que contém os gráficos da evolução do produto nas propostas de redução da tributação sobre a produção e do custo do capital de terceiros. Em ambos os casos, o custo de se permanecer formal cai, uma vez que as firmas precisarão gastar menos com o governo ou com os bancos. Por sua vez, o custo relativo da informalidade sobe, já que ficar formal ficou menos oneroso. Assim, ocorre contração do setor informal, acompanhada de crescimento do setor formal, estimulado tanto pelo crescimento das empresas que já estavam nessa situação, e que tiveram seus custos reduzidos, quanto pela migração das informais. $\mathrm{O}$ 


\section{Figura 1_Evolução da trajetória do produto}

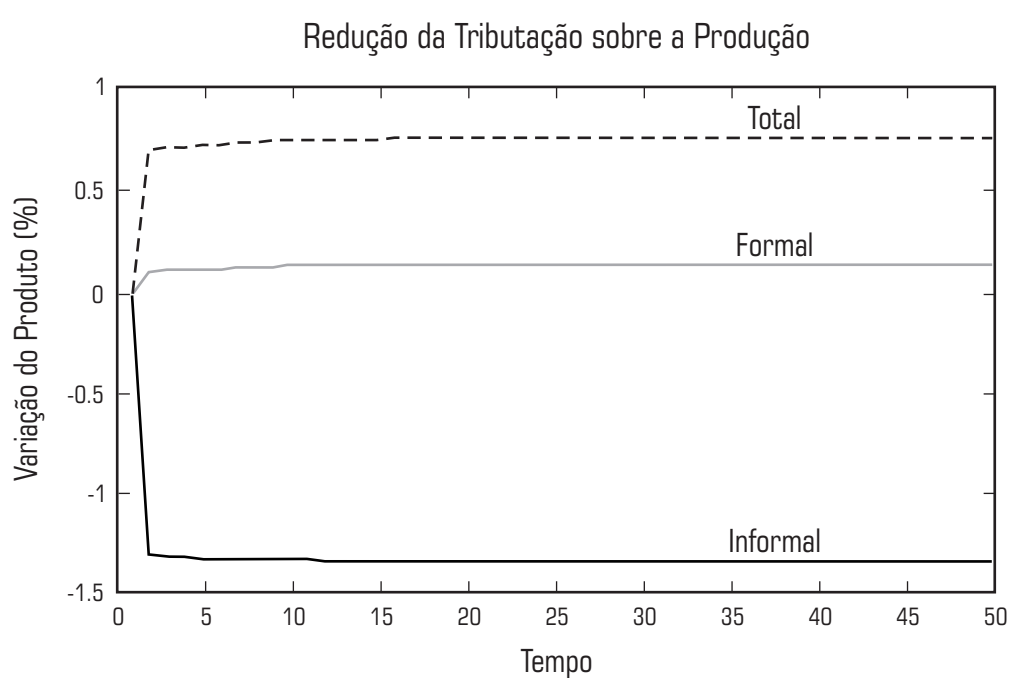

Redução do Custo do Capital de Terceiros

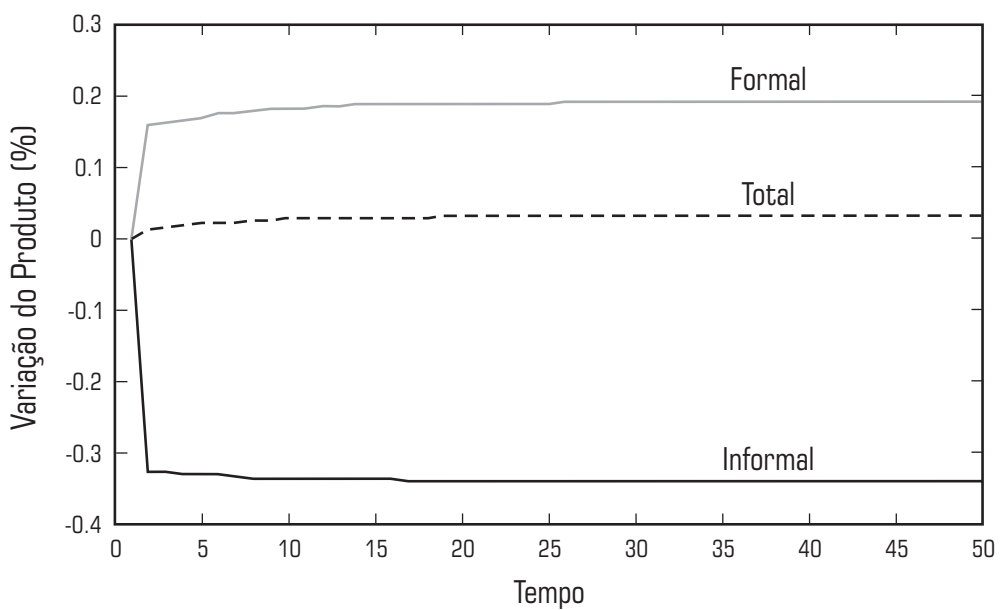

Fonte: Elaboração própria. 
produto total cresce pelo aumento do setor mais produtivo. O efeito é menor na redução do custo do crédito, porque esse ainda tem peso pequeno em relação ao estoque de capital das firmas formais, de maneira que o benefício é reduzido.

\section{2_ Emprego}

Quanto ao emprego, os resultados de estado estacionário estão na Tabela 2.

Os resultados relativos ao emprego estão bastante próximos ao do produto, exceto pelo emprego total. Notase que, ao contrário do que ocorreu com o produto total da economia, que cresceu na maioria das propostas, aqui o emprego total cai em todas as simulações. Isso ocorre porque as empresas, ao migraram do setor informal para o formal diante dos incentivos oferecidos, passam a ter acesso a tecnologias mais produ- tivas, o que reduz a sua necessidade de mão de obra vis-à-vis à necessidade que tinham na época da informalidade. As firmas conseguem produzir mais com menos trabalhadores na formalidade do que faziam quando eram informais. Assim, nem todos os trabalhadores das firmas informais que migraram mantêm o seu emprego, daí a redução no emprego total.

Percebe-se, mais uma vez, que o grande vilão da informalidade é a carga tributária, e que medidas coercitivas, embora ajudem a melhorar a situação, não são a melhor opção. Quanto à trajetória dinâmica do emprego, os gráficos da Figura 2 trazem os resultados obtidos com as simulações da redução da tributação sobre a produção e a redução do custo do capital de terceiros.

Tahela 2_Variação do emprego

\begin{tabular}{|c|c|c|c|}
\hline \multirow{2}{*}{ Propostas } & \multicolumn{3}{|c|}{ Variação } \\
\hline & Informal & Formal & Total \\
\hline Redução da Tributação sobre a Produção & $-1,32$ & 1,05 & $-0,36$ \\
\hline Redução da Tributação sobre a Renda do Capital & $-0,44$ & 0,30 & $-0,14$ \\
\hline Redução da Tributação sobre a Renda do Trabalho & $-1,26$ & 1,11 & $-0,30$ \\
\hline Aumento do Custo da Informalidade & $-0,74$ & 0,44 & $-0,26$ \\
\hline Redução do Custo do Crédito & $-0,33$ & 0,19 & $-0,12$ \\
\hline Aumento da Oferta de Crédito & $-0,08$ & 0,05 & $-0,02$ \\
\hline
\end{tabular}

Fonte: Elaboração própria. 


\section{Figura 2_Evolução da trajetória do produto}

Redução da Tributação sobre a Produção

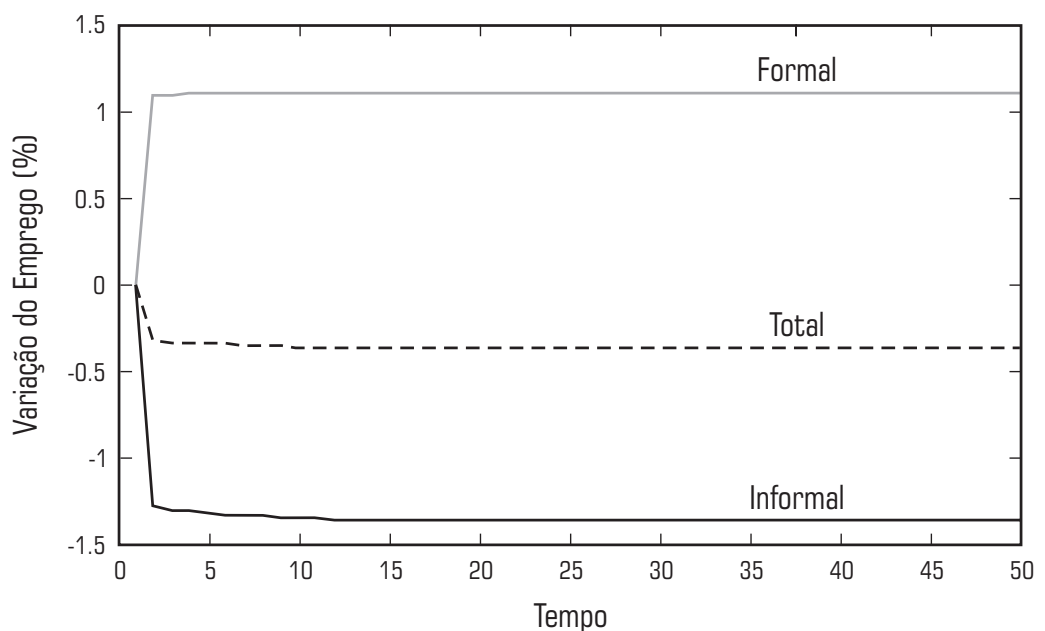

Redução do Custo do Capital de Terceiros

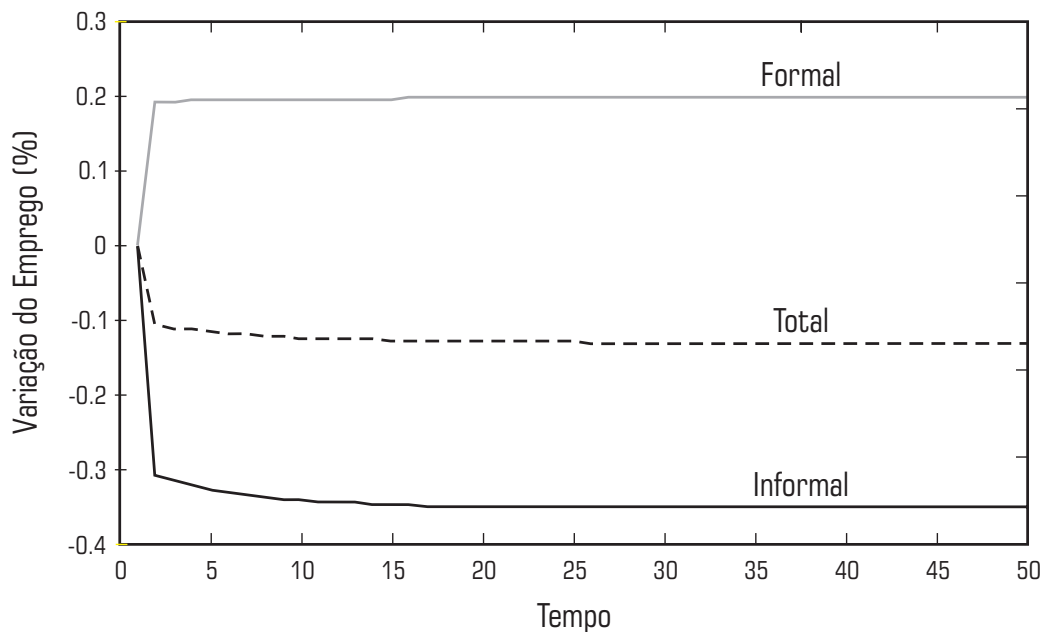

Fonte: Elaboração própria. 


\section{3_Arrecadação}

Visto como evolui a trajetória das duas variáveis mais importantes do trabalho, resta verificar como se comporta a arrecadação, principalmente em razão dos cortes de alíquotas tributárias. A Tabela 3 detalha os resultados:

O comportamento da arrecadação segue o comportamento do produto formal e o do emprego formal. A implantação das mudanças se traduz em pequeno aumento da arrecadação. Para as propostas que representam redução de alíquotas tributárias, o crescimento das receitas decorre do fato de que o aumento da base de cálculo foi de tal magnitude que suplantou a redução das alíquotas. Tais propostas apresentaram crescimento razoável no produto, emprego e estoque de capital formal. Para entender essa aparente contradição (redução da alíquo- ta versus aumento da arrecadação), devese notar que, ao se reduzir, por exemplo, a tributação sobre a renda do trabalho, uma firma informal que se torne formal passa a pagar tributos sobre os três fatos geradores de tributos, elevando a arrecadação. O segredo é a incorporação de firmas informais ao universo de contribuintes, aumentando significativamente as bases de cálculo dos tributos.

A redução dos custos de crédito também permite um incremento nas receitas. Ao contrário das propostas de tributação, nesse caso não há redução de quaisquer alíquotas, mas o crescimento das bases de cálculo é limitado pela pequena influência do crédito na economia, embora a redução do custo do crédito tenha sido mais efetiva do que a redução da tributação do capital sob a ótica da arrecadação.

Tahela 3_Evolução da arrecadação (\%)

\begin{tabular}{|c|c|c|c|c|c|c|}
\hline Tempo & $\begin{array}{c}\text { Redução Trib. } \\
\text { Produção }\end{array}$ & $\begin{array}{c}\text { Reduçãa Trib. } \\
\text { Capital }\end{array}$ & $\begin{array}{c}\text { Redução Trib. } \\
\text { Trabalho }\end{array}$ & $\begin{array}{l}\text { Aumento Custo } \\
\text { Informalidade }\end{array}$ & $\begin{array}{c}\text { Redução Custo } \\
\text { Crédito }\end{array}$ & $\begin{array}{c}\text { Aumento Oferta } \\
\text { Crédito }\end{array}$ \\
\hline 0 & 0,00 & 0,00 & 0,00 & 0,00 & 0,00 & 0,00 \\
\hline 5 & 0,22 & 0,01 & 0,34 & 0,31 & 0,12 & 0,02 \\
\hline 10 & 0,24 & 0,03 & 0,33 & 0,28 & 0,13 & 0,00 \\
\hline 20 & 0,25 & 0,05 & 0,32 & 0,26 & 0,14 & 0,00 \\
\hline 30 & 0,25 & 0,05 & 0,32 & 0,25 & 0,14 & 0,00 \\
\hline 50 & 0,25 & 0,05 & 0,32 & 0,25 & 0,14 & 0,00 \\
\hline
\end{tabular}

Fonte: Elaboração própria. 
Em relação ao aumento do custo da informalidade, o efeito sobre a arrecadação é limitado às firmas que deixam de ser informais e passam para a formalidade e às receitas decorrentes do incremento das penalidades sobre as firmas informais. Não houve incentivos tributários e creditícios para as firmas que já eram formais, como nos casos anteriores, proporcionando, no entanto, ganhos razoáveis de receitas.

\section{7_Conclusão}

O objetivo principal deste trabalho foi construir um arcabouço teórico que permitisse avaliar os impactos diretos e indiretos de políticas de combate à informalidade dentro de um contexto de equilíbrio geral. Optou-se, assim, por um modelo de equilíbrio geral computável baseado no modelo neoclássico de acumulação de capital, com utilização de tempo discreto e ênfase na dualidade formalinformal do setor produtivo, principalmente nos aspectos tributários e creditícios da economia.

Com o intuito de aplicar esse ferramental teórico para análise dos efeitos de políticas de redução da informalidade, construíram-se seis propostas alternativas: três delas baseadas na redução individual das alíquotas tributárias sobre cada uma das três bases de cálculo existentes no modelo (produto, renda do trabalho e renda do capital), outra fundada na redução do custo do capital de terceiros, uma quinta contemplando o incremento na disponibilidade do crédito, e uma última focada no aumento das penalidades e no custo de se manter informal.

Os resultados mostraram que a redução da carga tributária, principalmente no produto e no rendimento do trabalho, teria um efeito intenso na diminuição da informalidade na economia brasileira. Assim, se o objetivo de determinada política é reduzir a informalidade tanto na produção quanto no emprego, a redução na tributação sobre o produto, dada a grande distorção que traz para a economia, e também sobre o trabalho, tendo em vista a elevada alíquota e a maior intensidade do uso do trabalho nas empresas informais, é considerada uma boa alternativa a ser adotada. Isso porque reduziria mais que proporcionalmente o peso da informalidade na economia, trazendo, em conjunto, ganhos expressivos de produção e de emprego para o setor formal, além de expressivo crescimento do produto total da economia. 
A outra proposta de redução da tributação, o aumento do custo de permanecer informal e a redução do custo do crédito também apresentaram bons resultados, embora inferiores às duas já analisadas. Já a expansão do crédito teve pouco efeito, uma vez que os empréstimos são de relevância muito inferior ao estoque de capital alugado das famílias, o que reduz o alcance de políticas de crédito orientadas à redução da informalidade.

Quanto aos aspectos relativos às finanças públicas, o trabalho sugere que, mesmo com a redução das alíquotas tributárias, não haveria diminuição de arrecadação ao longo de todo o período. Isso se deve ao forte crescimento das bases tributáveis, que compensariam a perda da arrecadação oriunda da redução das alíquotas.

Trabalhos futuros poderão refinar a abordagem teórica aqui apresentada, de forma a avançar na modelagem dual formal-informal, maior detalhamento nas relações do mercado de trabalho e questões relacionadas ao produto setorial. 
Referência bibliográficas

ALTIG, D.; AUERBACH, A.; KOTLIKOFF, L.; SMETTTERS, K.; WALLISER, J. Simulating fundamental tax reform in the United States. The American Economic Review, v. 91, n. 3, p. $574-595,2001$

AMARAL, P.; QUINTIN, E. A competitive model of the informal sector. Journal of Monetary Economics, v. 53 , n. 7 , p. $1541-1553,2006$.

AGÊNCIA NACIONAL

DE ENERGIA ELÉTRICA

(ANEEL). Nota Técnica SRE/

ANEEL 097/2001.

ANTUNES, A.; CAVALCANTI,

T. Corruption, credit market imperfections and economic development. The Quarterly Review of Economics and Finance, v. 43, p. 627-642, 2003.

ANTUNES, A.; CAVALCANTI,

T. Start up costs, limited

enforcement, and the hidden economy. European Economic

Review, v. 51, n. 1 ,

p. 203-224, 2007.

ARAÚJO, C. H. V.; FERREIRA, P. C. Reforma tributária, efeitos alocativos e impactos de bemestar. Revista Brasileira de Economia, Rio de Janeiro, v. 53, n. 2, 1999.
AUERBACH, A.; KOTLIKOFF, L. Dynamic Fiscal Policy. Cambridge University Press, 1987.

\section{BANCO CENTRAL DO}

BRASIL. Relatório Anual 2004.

Banco Central do Brasil, Brasília.

Disponível em: <http://www.bcb. gov.br/?BOLETIM2004>. Acesso em: $12 / 05 / 2008$.

CARNEIRO, F. G. The changing informal labour market in Brazil: cyclicality versus excessive intervention. LABOUR. Review of Labour Economics and Industrial Relations, v. 11, n. 1, p. 1-22, 1997.

DE SOTO, H. Os mistérios do capital. Rio de Janeiro:

Record, 2001.

FERNANDES, R.; GREMAUD, A. P.; NARITA, R. T. Estrutura tributária e formalização da economia: simulando diferentes alternativas para o Brasil. In: ENCONTRO DA SOCIEDADE BRASILEIRA DE ECONOMETRIA, 29., 2004, João Pessoa. Anais... João Pessoa, 2004. p. 230-256.

FULLERTON, D. On the possibility of an inverse relationship between tax rates and government revenues. Journal of Public Economics, v. 19, p. 3-22, 1982.
INSTITUTO BRASILEIRO DE GEOGRAFIA E ESTATÍSTICA - IBGE. Sistema de Contas Nacionais 2000-2005. Rio de Janeiro, 2007. $72 \mathrm{p}$.

\section{JOHNSON, S.; KAUFMANN,} D.; ZOIDO-LOBATÓN. P.

Regulatory discretion and the unofficial economy. The American Economic Review, v. 88 n. 2 p. 387-392, 1998a.

JOHNSON, S.; KAUFMANN, D.; ZOIDO-LOBATÓN. P.

Corruption, public finances and the unofficial economy. The World Bank discussion paper, 1998b.

LOAYZA, N. A. The economics of the informal sector: a simple model and some empirical evidence from Latin America. Policy Research Working Paper, The World Bank, 1997.

MCKINSEY \& COMPANY.

Eliminando as barreiras ao crescimento econômico e à economia formal no Brasil. 2004. Mimeo.

NAME, R.; BUGARIN, M.

Fatores determinantes e evolução da economia submersa no Brasil. Estudos Econômicos, São Paulo, v. 33 , n. 2, 2003
PAES, N.; BUGARIN, M. Reforma tributária: impactos alocativos sobre o bem-estar e a progressividade. Revista Brasileira de Economia, Rio de Janeiro, v. 60, n. $3,2006$.

PASTORE, J. Como reduzir a informalidade? Mercado de Trabalbo: conjuntura e análise, v. 5, n. 14, p. $9-11$, out. 2000 .

PIGGOT, J.; WHALLEY, J. VAT base broadening, self supply, and the informal sector. The American Economic Review, v. 91, n. 4, p. 1084-1094, 2001.

PINHEIRO, V. C.; MIRANDA R. M. B. O perfil dos não contribuintes da Previdência Social. Informe da Previdência Social, mar. 2000.

RAMOS, L. A evolução da informalidade no Brasil metropolitano: 1991-2001. IPEA, 2002. (Texto para Discussão, 914).

REIS, M. C.; ULYSSEA, G. Cunba fiscal, informalidade e crescimento: algumas questões e propostas de políticas. IPEA, 2005. (Texto para Discussão, 1068). 
SCHNEIDER, F. The increase of the size of the informal economy of 18 OECD countries: some preliminary explanations. Paper presented at the Annual Public Choice Meeting, March 10-12, 2000, Charleston, S. C., 2000.

SCHNEIDER, F. Size and measurement of the informal economy in 110 countries around the world. Workshop of Australian National Tax Centre, ANU,

Canberra, Australia, July 17, 2002.

SCHNEIDER, F.; ENSTE, D. H. Shadow economies: size, causes, and consequences. Journal of

Economic Literature, v. 38, p. $77-114,2000$.

SERVIÇO BRASILEIRO DE APOIO ÀS MICRO E PEQUENAS EMPRESAS

- SEBRAE. Financiamento das MPEs no Estado de São Paulo. São Paulo, 2004. Disponível em: <http://www.sebraesp.com.br// principal/conhecendo $\% 20 \mathrm{a} \% 20$ mpe/estudos $\% 20$ temáticos/ financiamentompessp.aspx\#>. Acesso em: 16/07/2008.

\section{SECRETARIA DA RECEITA}

FEDERAL (SRF). Resultado da Fiscalização 2004. Receita Federal do Brasil, Brasília. Disponível em: $<$ http://www.receita.fazenda.gov. br>. Acesso em: 26/07/2008.

SHEPHERD, G.; HOLDEN, P. An assessment of the private sector in Brazil. Washington, DC: World Bank, 1993. Mimeo.
SHOVEN, J. E.; WHALLEY, J. Applying general equilibrium. Cambridge University Press, Cambridge, 1992.

STRAUB, S. Informal sector: the credit market channel. Journal of Development Economics, v. 78, n. 2, p. 299-321, 2005.

TANZI, V. Uses and abuses of estimates of the underground economy. The Economic Journal, v. 109, n. 456, p. 338-340, 1999.

Este trabalho contou com o
financiamento do Conselho Nacional
de Pesquisa Cientifica - CNPq no
ambito do Edital n. 50/2006.
$\vdots$
$\vdots$
e-mail de contato do autor:
$\vdots$
nppes @gmail.com
$\vdots$
artigo recebido em maio de 2009;
aprovado em fevereiro de 2010.


Apêndice

Aqui, $\beta$ denota o fator de desconto intertemporal, $\phi$ é o peso do consumo na função utilidade, $c_{t}$ é o consumo da família no tempo $t$ e $h_{t}$ são as horas trabalhadas pela família no tempo $t$.

$c_{t}-T_{t}+I_{t} \leq w_{t} h_{t}+r_{t} k_{t}+\prod_{F t}$

$k_{t+1}=(1-\delta) k_{t}+I_{t}$

Além disso, impomos que $\mathrm{k}_{\mathrm{t}}>0$. $\mathrm{Na}$ expressão acima, Tt é a transferência governamental recebida pela família no tempo t, e kt é o estoque de capital em t. O salário wt e a taxa de juros rt, bem como os lucros da firma formal $(\pi \mathrm{Ft})$ serão obtidos da condição de maximização do lucro das firmas representativas.

As famílias possuem uma dotação inicial de estoque de capital $\mathrm{k}_{0}$ que tomam como condição inicial. Ao se re- solver o problema de maximização, obtemos as sequências ótimas de consumo e horas de trabalho para as famílias:

$\mathrm{h}_{\mathrm{t}}=1-\frac{(1-\phi) \mathrm{c}_{\mathrm{t}}}{\phi \mathrm{w}_{\mathrm{t}}}$
$\mathrm{c}_{\mathrm{t}+1}=\beta\left(\mathrm{r}_{\mathrm{t}+1}+1-\delta\right) \mathrm{c}_{\mathrm{t}}$

A sequência ótima para o estoque de crapital no período seguinte é obtida a partir da substituição das duas equações acima na restrição orçamentária.

\section{Firmas}

Como a firma informal é competitiva, toma salários e remuneração do capital como dados e escolhe o nível ótimo de capital e horas de trabalho, resolvendo o seguinte problema de maximização:

$$
\begin{aligned}
\pi_{l t}= & \max _{h_{t t}}(1-p) k_{l t}^{\alpha_{l}} h_{l t}^{1-\alpha_{l}}- \\
& -(1+p) w_{t} h_{l t}-(1+p) r_{t} k_{l t}
\end{aligned}
$$

O parâmetro p representa o custo da sonegação para as empresas informais, em percentual da base de cálculo do imposto. Ele capta a probabilidade de a empresa ser descoberta pela fiscalização, o percentual da multa a ser aplicada e a eficiência do sistema judiciário em fazer cumprir as penalidades. Resolvendo o problema (6), obtemos: 


$$
\begin{aligned}
& w_{t}=\frac{(1-p)\left(1-\alpha_{I}\right) k_{l t}^{\alpha} h_{t t}^{-\alpha_{l}}}{(1+p)} \\
& r_{t}=\frac{(1-p) \alpha_{I} k_{l t}^{\alpha_{l}-1} h_{l t}^{1-\alpha l}}{(1+p)}
\end{aligned}
$$

Já a firma formal, que pode recorrer ao mercado de crédito de forma a aumentar a sua produção, inicialmente escolhe a quantidade ótima de trabalho a ser contratada para um dado estoque de capital k, resolvendo o problema de maximização de lucros:

$$
\begin{aligned}
\pi_{\mathrm{Ft}}\left(\mathrm{k}_{\mathrm{F}}, \mathrm{W}\right)= & \max _{\mathrm{hFt}}\left(1-\tau_{\mathrm{pt}}\right)\left(\mathrm{k}_{\mathrm{Ft}}+\mathrm{b}_{\mathrm{t}}\right)^{\alpha \mathrm{F}} \\
& \left(\mathrm{Ah}_{\mathrm{Ft}}\right)^{\gamma \mathrm{F}}-\left(1+\tau_{\mathrm{ht}}\right) \mathrm{W}_{\mathrm{t}} \mathrm{h}_{\mathrm{Ft}}
\end{aligned}
$$

Aqui, $\tau_{\mathrm{pt}}$ é a alíquota tributária sobre a produção, $\tau_{h t}$ é a alíquota tributária sobre a renda do trabalho, e $b_{t}$ representa a demanda por crédito que será suprida por financiadores externos. Observa-se que a função de produção da firma formal conta com um fator exógeno de produtividade, A, que retrata a conhecida maior eficiência da produtividade do trabalho do setor formal em relação ao informal. Esta diferença de produtividade decorre de uma série de fatores, como, por exemplo: o acesso a tecnologias mais produtivas, incluindo melhores máquinas e equipamentos; a presença de trabalhadores mais qualificados; e os ga-

nhos de escala (firmas formais tendem a ser consideravelmente maiores do que as informais).

Resolvendo o problema acima, encontramos a demanda ótima de trabalho da firma formal e a demanda global de trabalho:

$$
\begin{aligned}
& h_{\mathrm{Ft}}=\left(\frac{\gamma_{\mathrm{F}} \mathrm{A}^{\gamma_{\mathrm{F}}}\left(1-\tau_{\mathrm{pt}}\right)\left(\mathrm{k}_{\mathrm{Ft}}+\mathrm{b}_{\mathrm{t}}\right)^{\alpha_{\mathrm{F}}}}{\left(1+\tau_{\mathrm{ht}}\right) \mathrm{W}_{\mathrm{t}}}\right)^{1 / 1-\gamma_{\mathrm{F}}} \\
& \mathrm{h}_{\mathrm{t}}=\mathrm{h}_{\mathrm{Ft}}+\mathrm{h}_{\mathrm{It}}
\end{aligned}
$$

Reescrevendo a equação (9), considerando o nível ótimo das horas de trabalho, encontra-se:

$\pi_{F t}\left(k_{F}, w\right)=\left\{\left[\left(1-\tau_{p t}\right)\left(k_{F t}+b_{t}\right)^{\alpha_{F}}\right]^{1 / 1 / \gamma_{F}}\left(1-\gamma_{F}\right)\left(\frac{A \gamma_{F}}{\left(1+\tau_{h t}\right) w_{t}}\right)^{\gamma_{F} / 1-\gamma_{F}}\right\}$

Em seguida, as firmas formais podem escolher o nível ótimo de capital, de acordo com a seguinte equação:

$\max \pi_{F t}\left(\left(k_{F}+b\right), w, r\right)\left(1-\tau_{k t}\right)-\left(r_{t}-\theta\right) b_{t}-r_{t} k_{F t}$

onde $\theta$ é a diferença entre o custo de capital de terceiros e o custo do capital próprio. Neste modelo, a oferta de crédito é limitada a uma parte do capital físico (aquela parte que pode ser aceita como garantia para o fornecimento de empréstimo). ${ }^{11}$ As firmas informais não podem tomar emprestado, uma vez que
${ }_{11}$ O restante do capital, apesar de existir no mundo físico, não tem valor financeiro e não pode ser usado para a tomada de crédito, e daí a sua denominação de "capital latente”, criada por De Soto, em seu conhecido livro O mistério do capital (2001). 
o capital por elas utilizado não é aceito como garantia ("capital latente"), enquanto as formais podem expandir sua produção, aproximando-se do nível ótimo de capital, já que os financiadores externos aceitam parte do capital que elas utilizam como garantia.

Assim, a oferta de crédito será dada por $b_{t}=\eta * k_{\mathrm{Ft}}$, sendo $\eta$ o percentual do capital das firmas formais que pode ser aceito como colateral. Desta maneira, introduzindo a restrição ao crédito no problema de maximização das firmas, (13), determina-se a demanda de capital das firmas formais a ser suprida pelas famílias:

$$
\begin{aligned}
\mathrm{k}_{\mathrm{Ft}}(\mathrm{W}, \mathrm{r})= & \left\{\frac{\alpha_{\mathrm{F}}\left(1-\tau_{\mathrm{kt}}\right)}{\mathrm{r}_{\mathrm{t}}+\left(\mathrm{r}_{\mathrm{t}}-\theta\right) \eta}(1+\eta)^{\alpha_{\mathrm{F}} / 1-\gamma_{\mathrm{F}}}\left(1-\tau_{\mathrm{pt}}\right)^{1 / 1-\gamma_{\mathrm{F}}}\right. \\
& \left.\left(\frac{\mathrm{A} \gamma_{\mathrm{F}}}{\left(1+\tau_{\mathrm{ht}}\right) \mathrm{W}_{\mathrm{t}}}\right)^{\gamma_{\mathrm{F}} / 1-\gamma_{\mathrm{F}}}\right\}^{1-\gamma_{\mathrm{F}} / 1-\alpha_{\mathrm{F}}-\gamma_{\mathrm{F}}}
\end{aligned}
$$

De forma que o empréstimo externo será de $\eta * \mathrm{k}_{\mathrm{Ft}}$. A oferta total de capital pelas famílias será dada por:

$$
k_{t}=k_{F t}+k_{I t}
$$

\section{Governo}

O governo arrecada impostos das firmas formais e multas das informais para financiar seus gastos e transferências, mantendo um orçamento equilibrado em cada período. Isso significa que o conjunto das sequências relativo às variáveis e parâmetros fiscais $\left\{T_{t}, g_{t}, \tau_{h t}, \tau_{p t}, \tau_{k t}\right\}$ é dado por uma decisão política exógena. Portanto, a restrição orçamentária do governo é dada pela expressão:

$$
\Gamma_{t}=T_{t}+g_{t}
$$

Em que $\Gamma_{t}$ corresponde à arrecadação tributária, e $\mathrm{T}_{\text {t }}$, o total de transferências, ambos do período t.

\section{Descrição do equilíbrio}

$\mathrm{Na}$ economia artificial acima descrita, as famílias escolherão as sequências $\left\{c_{t}\right.$ $\left., \mathrm{h}_{\mathrm{t}}, \mathrm{k}_{\mathrm{t}}\right\}$ kque maximizam sua utilidade sujeitas à restrição orçamentária. Por sua vez, a firma representativa formal escolh $\left\{\mathrm{k}_{\mathrm{Ft}}, \mathrm{h}_{\mathrm{Ft}}\right\}$ e a informal, $\left\{\mathrm{k}_{\mathrm{It}}, \mathrm{h}_{\mathrm{It}}\right\}$ de maneira a maximizar seus lucros.

\section{Definição 1}

Uma politica fiscal plausivel do governo é uma sequência de alíquotas tributárias, de despesas e de transferências que satisfazem a restrição orçamentária do governo (18).

\section{Definição 2}

O sistema de preços da economia é formado pelo conjunto de sequências de variáveis reais $\left\{\mathrm{w}_{\mathrm{t}}, \mathrm{r}_{\mathrm{t}}\right\}$. 


\section{Definição 3}

Uma alocação plausivel é uma sequência de consumo, horas trabalhadas e estoque de capital físico $\left\{c_{t}, h_{t}, k_{t}\right\}$ que satisfaz a restrição agregada.

$c_{t}+\left[k_{t+1}-(1-\delta) k_{t}\right]+g_{t}+\left(r_{t}-\theta\right) b_{t}=$

$=\left(k_{F t}+b_{t}\right)^{\alpha_{F}}\left(A h_{F t}\right)^{\gamma_{F}}+k_{l t}^{\alpha} h_{l t}^{1-\alpha_{1}}$

\section{Definição 4}

Um equilibrio competitivo com tributos distorcivos é composto de uma política fiscal compatível com a restrição orçamentária do governo, uma alocação plausível e um sistema de preços tal que, dado o sistema de preços e a política fiscal, a alocação resolve os problemas das firmas e da família.

Para o cálculo do equilíbrio, devese resolver o sistema de equações de diferenças não lineares composto de (4), (5), (7), (8), (10), (11), (14), (15), (16) e (17), dado $\mathrm{k}_{0}$.

É possível simplificar o sistema de equações de forma a se trabalhar apenas com quatro equações em cada instante t. Assim, substituindo (4), (7), (8), (10), (14) e (17), obtém-se:

$$
\begin{aligned}
& c_{t}+\left[k_{t+1}-(1-\delta) k_{t}\right]+g_{t}+\left(\alpha_{1} \frac{(1-p)}{(1+p)} k_{i t}^{\alpha_{1}-1} h_{i t}^{1-\alpha_{1}}-\theta\right) \eta . \\
& \left\{\frac{\alpha_{\mathrm{F}}\left(1-\tau_{\mathrm{kt}}\right)(1-\eta)^{\alpha_{\mathrm{F}} / 1-\gamma_{\mathrm{F}}}\left(1-\tau_{\mathrm{pt}}\right)^{1 / 1 / \gamma_{\mathrm{F}}}}{\alpha_{1} \frac{(1-p)}{(1+p)} k_{\mathrm{it}}^{\alpha_{1}-1} h_{\mathrm{it}}^{1-\alpha_{\mathrm{I}}}(1+\eta)-\theta \eta} \cdot\left(\frac{\mathrm{A} \gamma_{\mathrm{F}}}{\left(1+\tau_{\mathrm{ht}}\right)\left(1-\alpha_{\mathrm{I}}\right) \frac{(1-\mathrm{p})}{(1+\mathrm{p})} \mathrm{k}_{\mathrm{it}}^{\alpha_{\mathrm{it}}} \mathrm{h}_{\mathrm{it}}^{-\alpha_{\mathrm{i}}}}\right)^{\gamma_{\mathrm{F}} / 1-\gamma_{\mathrm{F}}}\right\}^{1-\gamma_{\mathrm{F}} / 1-\gamma_{\mathrm{F}}}= \\
& =\left\{\left[\frac{\alpha_{\mathrm{F}}(1+\eta)\left(1-\tau_{\mathrm{kt}}\right)}{\alpha_{\mathrm{I}} \frac{(1-\mathrm{p})}{(1+\mathrm{p})} \mathrm{k}_{\mathrm{it}}^{\alpha_{\mathrm{t}}-1} \mathrm{~h}_{\mathrm{it}}^{1-\alpha_{\mathrm{I}}}(1+\eta)-\theta \eta}\right]^{\alpha_{\mathrm{F}}}\left(1-\tau_{\mathrm{pt}}\right)^{\alpha_{\mathrm{F}}+\gamma_{\mathrm{F}}} \cdot\left(\frac{\mathrm{A} \gamma_{\mathrm{F}}}{\left(1+\tau_{\mathrm{ht}}\right)\left(1-\alpha_{\mathrm{l}}\right) \frac{(1-\mathrm{p})}{(1+\mathrm{p})} \mathrm{k}_{\mathrm{it}}^{\alpha_{\mathrm{t}}} h_{\mathrm{it}}^{-\alpha_{\mathrm{f}}}}\right)^{\gamma_{\mathrm{FF}}}\right\}^{1 / 1-\alpha_{\mathrm{F}}-\gamma_{\mathrm{F}}}+\mathrm{k}_{\mathrm{lt}}^{\alpha_{\mathrm{t}}} h_{\mathrm{lt}}^{1-\alpha_{\mathrm{l}}}
\end{aligned}
$$

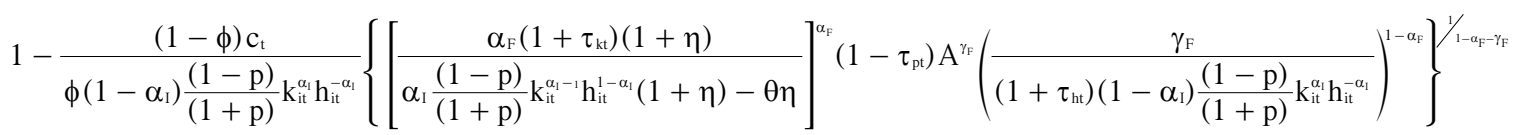

$$
\begin{aligned}
& \mathrm{c}_{\mathrm{t}+1}=\beta\left(\alpha_{1} \frac{(1-\mathrm{p})}{(1+\mathrm{p})} \mathrm{k}_{\mathrm{it}+1}^{\alpha_{\alpha}-1} \mathrm{~h}_{\mathrm{it}+1}^{1-\alpha_{1}}+1-\delta\right) \mathrm{c}_{\mathrm{t}}
\end{aligned}
$$

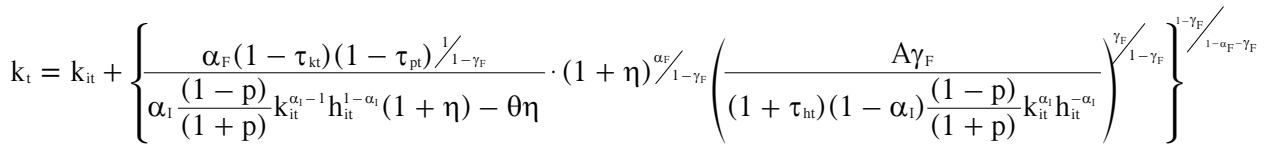




\section{Solução do modelo}

Usando o algoritmo de Broyden para solução de sistemas de equações não lineares, determinam-se as trajetórias $\left\{\mathrm{c}_{\mathrm{t}}\right\}_{0}^{\mathrm{S}},\left\{\mathrm{k}_{\mathrm{t}}\right\}_{0}^{\mathrm{S}},\left\{\mathrm{k}_{\mathrm{it}}\right\}_{0}^{\mathrm{S}},\left\{\mathrm{h}_{\mathrm{it}}\right\}_{0}^{\mathrm{s}}$ e obtêm-se as trajetórias das outras variáveis com as demais equações. 
